\title{
Priors in quantum Bayesian inference
}

\author{
Christopher A. Fuchs* and Rüdiger Schack ${ }^{\dagger}$ \\ ${ }^{*}$ Perimeter Institute for Theoretical Physics, 31 Caroline Street North, Waterloo, Ontario, Canada \\ N2L $2 Y 5$ \\ ${ }^{\dagger}$ Department of Mathematics, Royal Holloway, University of London, Egham, Surrey TW20 OEX, \\ $U K$
}

\begin{abstract}
In quantum Bayesian inference problems, any conclusions drawn from a finite number of measurements depend not only on the outcomes of the measurements but also on a prior. Here we show that, in general, the prior remains important even in the limit of an infinite number of measurements. We illustrate this point with several examples where two priors lead to very different conclusions given the same measurement data.
\end{abstract}

Keywords: Bayesian probability, quantum inference, quantum measurement

PACS: 03.65.Wj

\section{INTRODUCTION}

This paper addresses the problem of inference in quantum mechanics in a very general setting. Consider a sequence of quantum systems, each with Hilbert space $\mathscr{H}$ which, for simplicity, is assumed to have finite dimension. Now some of the systems are measured. What conclusions can be drawn from the measurement outcomes?

It is often useful to consider less general situations. One of the most studied quantum inference problems is quantum state estimation, which is frequently described as follows [1]. Each system is assumed to have the same unknown state $\sigma$, where $\sigma$ is a density operator on $\mathscr{H}$. A sequence of appropriately chosen measurements is then used to determine $\sigma$. The most general single-system measurement is described by a POVM, which is a collection of positive operators, $\left\{E_{1}, \ldots, E_{r}\right\}$, acting on $\mathscr{H}$, such that $\sum_{k} E_{k}$ is the identity operator. The index $k \in\{1, \ldots, r\}$ labels the possible measurement outcomes. If the state of the system is $\rho$, the probability of obtaining outcome $k$ is given by $\operatorname{tr}\left(\rho E_{k}\right)$.

The Bayesian solution to the quantum state estimation problem is straightforward. Its starting point is a prior probability distribution $p_{\text {prior }}(\rho)$ on the set of density operators on $\mathscr{H}$. Assume that the POVM $\left\{E_{k}\right\}$ is measured for a single system. If the outcome of the measurement is $k$, the distribution $p_{\text {prior }}(\rho)$ is updated using a quantum version of Bayes's rule [2, 3], resulting in the posterior distribution

$$
p_{\text {posterior }}(\rho)=\frac{p_{\text {prior }}(\rho) \operatorname{tr}\left(\rho E_{k}\right)}{\int d \rho p_{\text {prior }}(\rho) \operatorname{tr}\left(\rho E_{k}\right)} .
$$

This process is iterated, each time using a fresh copy of the system and possibly a different measurement, and each time setting $p_{\text {prior }}(\rho)$ equal to the previously obtained $p_{\text {posterior }}(\rho)$. Given some mild conditions on the original prior distribution, and assuming that the measurements are appropriately chosen, it can be shown that the iterated distribution approaches a delta distribution centered at the unknown state $\sigma$. For instance, 
this convergence holds if the original $p_{\text {prior }}(\rho)$ is nonzero for all $\rho$ and the measured POVM is informationally complete [4].

In practice, the iteration will have to stop after a finite number of measurements. The posterior distribution will then generally depend on the initial prior distribution. From a Bayesian perspective, this dependence on the prior distribution is unavoidable. Finding the appropriate mathematical form of the prior distribution is a central part of the Bayesian approach to quantum state estimation.

There are a number of (non-Bayesian) state estimation methods that attempt to circumvent the dependence of the estimate on the prior distribution [1]. An important example is maximum likelihood estimation. We are not going to discuss non-Bayesian methods any further in this paper.

In the next section, we will show that quantum state estimation as described above is a special case of a far more general quantum inference scenario in which priors retain their importance even in the limit of an infinite number of measurements. This is followed by a section illustrating this point with some examples. The paper concludes with a brief discussion.

\section{GENERAL QUANTUM INFERENCE PROBLEM}

There is something peculiar about the quantum Bayes rule (1). Since a density operator $\rho$ encodes the probabilities for outcomes of quantum measurements, the rule (1) mixes two kinds of probabilities, namely the "classical" probabilities $p_{\text {prior }}$ and $p_{\text {posterior }}$ on the one hand, and the "quantum" probabilities encoded in $\rho$ on the other hand. We will now show that the rule (1) is a special case of a more general rule phrased entirely in terms of quantum states. It is therefore not necessary to make the distinction between two kinds of probability.

There is a general updating rule built into quantum theory. To describe a quantum measurement fully, one needs to provide, in addition to the POVM $\left\{E_{1}, \ldots, E_{r}\right\}$, a set of Kraus operators $A_{k j}$ such that $E_{k}=\sum_{j} A_{k j} A_{k j}^{\dagger}$ for $k=1, \ldots, r$ [5]. If the measurement gives outcome $k$ for a system in state $\rho$, the state of the system after the measurement will be

$$
\rho_{k}=\operatorname{tr}\left(\rho E_{k}\right)^{-1} \sum_{j} A_{k j} \rho A_{k j}^{\dagger} .
$$

This rule has an interpretation very similar to the Bayes rule. If we call $\rho$ the prior state and $\rho_{k}$ the posterior state, we see that the prior state is changed into the posterior state upon acquisition of the data $k$.

To see that the quantum Bayes rule (11) is a special case of the Kraus rule (2) [2], consider a sequence of quantum systems each with Hilbert space $\mathscr{H}$ as before. We define a prior on our sequence of systems as a sequence of $n$-system states $\rho_{\text {prior}}^{(n)}$, $n=1,2, \ldots$, where each $\rho_{\text {prior }}^{(n)}$ is a density operator on the $n$-fold tensor-product Hilbert space $\mathscr{H}^{\otimes n}=\mathscr{H} \otimes \cdots \otimes \mathscr{H}$, and where $\rho_{\text {prior }}^{(n)}=\operatorname{tr}_{n+1} \rho_{\text {prior }}^{(n+1)}$ for all $n \geq 1$. By $\operatorname{tr}_{n+1}$ we denote the trace over the $(n+1)$-th system. In words, each member of the sequence is obtained from the next by tracing over the additional system. 
Now assume that the first system is measured and an outcome $k$ is obtained. Applying the rule (2) we find, for any $n \geq 1$, that the state of the first $n$ systems after the measurement is

$$
\rho_{k}^{(n)}=\operatorname{tr}\left(\rho_{\text {prior }}^{(n)} E_{k}\right)^{-1} \sum_{j} A_{k j} \rho_{\text {prior }}^{(n)} A_{k j}^{\dagger},
$$

where it is understood that the operators $A_{k j}$ and $E_{k}$ act on the first system only. By tracing over the first system, we obtain what we call the posterior on our sequence of systems,

$$
\rho_{\text {posterior }}^{(n)}=\operatorname{tr}_{1} \rho_{k}^{(n+1)}(n=1,2, \ldots) .
$$

The posterior is again a sequence of states; its $n$-th member is obtained from the $(n+1)$ th member of the prior by measuring and then discarding the first system. The posterior has the property $\rho_{\text {posterior }}^{(n)}=\operatorname{tr}_{n+1} \rho_{\text {posterior }}^{(n+1)}$ for all $n \geq 1$ and thus has the form of a prior. We can therefore iterate the above procedure, each time setting the prior equal to the posterior obtained in the previous iteration.

Given the prior and the measurement data, the posterior is the unique sequence of states for the remaining (i.e., not yet measured) systems. In this sense the posterior constitutes the unique correct solution of the quantum inference problem. In particular, the one-system state $\rho_{\text {posterior }}^{(1)}$ is the marginal state for the first unmeasured system. This state is sometimes called the Bayesian mean estimator.

To recover the familiar rule (1), only one simple additional assumption has to made, namely that for any $n \geq 1$ the state $\rho_{\text {prior }}^{(n)}$ is symmetric under permutations of the $n$ systems. In this case we say that the prior is exchangeable [6]. Given this extra assumption, it is the content of the quantum de Finetti theorem [4, 6] that the prior can be written as

$$
\rho_{\text {prior }}^{(n)}=\int d \rho p_{\text {prior }}(\rho) \rho^{\otimes n} \quad(n=1,2, \ldots),
$$

where $p_{\text {prior }}(\rho)$ is a probability distribution on the space of single-system density operators, and $\rho^{\otimes n}$ is the $n$-fold tensor product $\rho \otimes \cdots \otimes \rho$. It is not difficult to establish [2] that a measurement on the first system with outcome $k$ will lead to the posterior

$$
\rho_{\text {posterior }}^{(n)}=\int d \rho p_{\text {posterior }}(\rho) \rho^{\otimes n}(n=1,2, \ldots),
$$

with $p_{\text {posterior }}(\rho)$ given by the rule (1).

As was pointed out in the introduction, in the limit of an infinite number of iterations, $p_{\text {posterior }}(\rho)$ typically approaches a delta function which is independent of the detailed functional form of $p_{\text {prior }}(\rho)$. This conclusion, however, depends crucially on the assumption that the prior is of the form (5), i.e., that the prior is exchangeable. In other words, even in the limit of an infinite number of iterations, conclusions depend on the prior. All we can say is that some details of the prior become irrelevant in this limit.

Exchangeable priors are an important class of priors that are used so frequently that it is sometimes overlooked that exchangeability is an assumption. Making this assumption is equivalent to choosing a prior from a restricted set. What we have therefore shown in this section is that conclusions drawn in Bayesian quantum inference situations generally 
depend on the prior as well as measurement data, even in the limit of an infinite number of measurements. In the next section, we illustrate this point with some examples.

\section{EXAMPLE PRIORS}

The first example is a sequence of qubits, i.e., two-dimensional quantum systems. We denote by $|0\rangle$ and $|1\rangle$ two orthogonal basis states and consider three priors.

Our first prior is exchangeable and given by

$$
\rho_{a}^{(n)}=\int d|\psi\rangle p_{a}(|\psi\rangle)(|\psi\rangle\langle\psi|)^{\otimes n} \quad(n=1,2, \ldots),
$$

where $d|\psi\rangle p_{a}(|\psi\rangle)$ is the Haar measure on the space of pure one-qubit states.

Our second prior is also exchangeable, but consists of a sequence of pure product states. It could be called a Rosenkrantz and Guildenstern prior [7]. This is the state one might assign to a quantum random number generator manufactured by a trusted company. It is given by

$$
\rho_{b}^{(n)}=\left(\frac{1}{2}(|0\rangle+|1\rangle)(\langle 0|+\langle 1|)\right)^{\otimes n} \quad(n=1,2, \ldots) .
$$

Our third prior is not exchangeable. We call it a counter-inductive prior [8] because it leads one to predict outcomes that are the opposite of what an argument by induction would suggest. In particular, we will see that updating this prior after a string of $m$ identical measurement outcomes, the probability for obtaining the opposite outcome in the next measurement approaches 1 as $m$ increases. The counter-inductive prior is given by

$$
\begin{aligned}
\rho_{c}^{(n)}= & \mathscr{N}\left[\sum _ { k = 1 } ^ { n - 1 } 2 ^ { - k ^ { 2 } } \left(|0\rangle\left\langle\left. 0\right|^{\otimes k} \otimes \mid 1\right\rangle\left\langle\left. 1\right|^{\otimes(n-k)}+\mid 1\right\rangle\left\langle\left. 1\right|^{\otimes k} \otimes \mid 0\right\rangle\left\langle\left. 0\right|^{\otimes(n-k)}\right)\right.\right. \\
& +\sum_{k=n}^{\infty} 2^{-k^{2}}\left(|0\rangle\left\langle\left. 0\right|^{\otimes n}+\mid 1\right\rangle\left\langle\left. 1\right|^{\otimes n}\right)\right] \quad(n=1,2, \ldots),
\end{aligned}
$$

where the normalization constant $\mathscr{N}$ is determined by the equation

$$
1=2 \mathscr{N} \sum_{k=1}^{\infty} 2^{-k^{2}} .
$$

It is not difficult to check that this sequence of states satisfies the defining condition of a prior, $\rho_{c}^{(n)}=\operatorname{tr}_{n+1} \rho_{c}^{(n+1)}$ for $n \geq 1$.

We now imagine that a sequence of von Neumann measurements in the $\{|0\rangle,|1\rangle\}$ basis is carried out and that each measurement produces the same outcome, 0 . The measurement data thus consist of a string of zeros. These data are used to update iteratively each of our three priors above. For each prior, we compute the marginal onesystem posteriors in the limit of an infinite number of iterations. 
For the two exchangeable priors, we obtain easily the limits

$$
\rho_{a}^{(1)} \rightarrow|0\rangle\langle 0| \quad \text { (number of iterations } \rightarrow \infty \text { ) }
$$

and

$$
\rho_{b}^{(1)} \rightarrow \frac{1}{2}(|0\rangle+|1\rangle)(\langle 0|+\langle 1|) \quad \text { (number of iterations } \rightarrow \infty \text { ). }
$$

To compute the limit for the counter-inductive prior $\rho_{c}^{(n)}$, we first compute the posterior after $m$ iterations, $\rho_{c, m}^{(n)}$. We find, for $n=1,2, \ldots$,

$\rho_{c, m}^{(n)}=\mathscr{N}_{m}\left(|1\rangle\left\langle\left. 1\right|^{\otimes n}+\sum_{k=1}^{n-1} 2^{-k(2 m+k)} \mid 0\right\rangle\left\langle\left. 0\right|^{\otimes k} \otimes \mid 1\right\rangle\left\langle\left. 1\right|^{\otimes(n-k)}+\sum_{k=n}^{\infty} 2^{-k(2 m+k)} \mid 0\right\rangle\left\langle\left. 0\right|^{\otimes n}\right)\right.$

where $\mathscr{N}_{m}$ is determined by the condition

$$
1=\mathscr{N}_{m} \sum_{k=0}^{\infty} 2^{-k(2 m+k)}
$$

implying that $\lim _{m \rightarrow \infty} \mathscr{N}_{m}=1$. Hence

$$
\rho_{c}^{(1)} \rightarrow|1\rangle\langle 1| \text { (number of iterations } \rightarrow \infty \text { ). }
$$

Clearly, the three priors lead to radically different conclusions for the same infinite sequence of data.

We now move on to our second example, where we compare two priors, again for a sequence of qubits. For our first prior, we choose a generic exchangeable prior,

$$
\rho_{d}^{(n)}=\int d \rho p_{d}(\rho) \rho^{\otimes n} \quad(n=1,2, \ldots),
$$

where $d \rho p_{d}(\rho)$ is a measure on one-qubit density operators, i.e., density operators on a two-dimensional Hilbert space, $\mathscr{H}$. We assume that $p_{d}(\rho)$ is nonzero for all $\rho$ on $\mathscr{H}$. This prior entails that there is no entanglement between the qubits.

By contrast, our second prior, though identical with our first on the single system marginals, does not rule out entanglement between pairs of qubits. For even numbers of systems, it is defined by

$$
\rho_{e}^{(2 n)}=\int d \sigma p_{e}(\sigma) \sigma^{\otimes n} \quad(n=1,2, \ldots),
$$

where $d \sigma p_{e}(\sigma)$ is a measure on the space of two-qubit density operators, i.e., density operators on the four-dimensional Hilbert space $\mathscr{H} \otimes \mathscr{H}$. For odd numbers of systems, the prior is defined by

$$
\rho_{e}^{(2 n-1)}=\operatorname{tr}_{2 n} \rho_{e}^{(2 n)} \quad(n=1,2, \ldots)
$$


Assume now that a sequence of informationally complete measurements is performed on the sequence of qubits. As before, the measurement data are used to update both our priors iteratively. We assume that the data are such that for the second prior, the marginal two-system posterior converges to an entangled two-qubit state, e.g., the maximally entangled state $\rho_{M E}=\frac{1}{2}(|00\rangle+|11\rangle)(\langle 00|+\langle 11|)$. We thus assume that

$$
\rho_{e}^{(2)} \rightarrow \rho_{M E} \quad \text { (number of iterations } \rightarrow \infty \text { ). }
$$

Since for the maximally entangled state, both marginal states are equal to the totally mixed state $\rho_{M}=\frac{1}{2}(|0\rangle\langle 0|+| 1\rangle\langle 1|)$, it follows that given the same data, the marginal one-system posterior for our first prior converges to $\rho_{M}$,

$$
\rho_{d}^{(1)} \rightarrow \rho_{M} \quad \text { (number of iterations } \rightarrow \infty \text { ), }
$$

and the marginal two-system posterior converges to

$$
\rho_{d}^{(2)} \rightarrow \rho_{M} \otimes \rho_{M} \quad \text { (number of iterations } \rightarrow \infty \text { ), }
$$

which is equal to the maximally mixed state of two qubits and, of course, not entangled.

Once more, we see that the same infinite sequence of data leads to radically different conclusions for the two priors.

\section{CONCLUSION}

The most general way in quantum mechanics for obtaining a quantum state from data is via the Kraus rule (2). It is clear that the quantum state obtained in this way generally depends on some prior state in addition to the data. In an earlier paper [9] we have established the general principle that a quantum state is never determined by measurement data alone. This is true even in state preparation, because the prepared state always depends on the prior quantum state of the preparation device [9].

What we have illustrated here it that this general principle continues to hold in situations where measurements are repeated many times. A quantum state is never determined by measurement data alone, even in the limit of infinitely many measurements.

\section{REFERENCES}

1. J. Řeháček, and M. G. A. Paris, editors, Quantum State Estimation, Springer Verlag, Berlin, 2004, Lecture Notes in Physics 649, and references therein.

2. T. A. Brun, C. M. Caves, and R. Schack, Phys. Rev. A 63, 042309.1-10 (2001).

3. J. M. Bernardo, and A. F. M. Smith, Bayesian Theory, Wiley, Chichester, 1994.

4. C. M. Caves, C. A. Fuchs, and R. Schack, J. Math. Phys. 43, 4537-4559 (2002).

5. K. Kraus, States, Effects, and Operations. Fundamental Notions of Quantum Theory, Springer Verlag, Berlin, 1983, Lecture Notes in Physics 190.

6. R. L. Hudson, and G. R. Moody, Z. Wahrscheinlichkeitstheorie verw. Geb. 33, 343-351 (1976).

7. T. Stoppard, Rosenkrantz and Guildenstern are Dead, Faber and Faber, London, 1967. In this play, Guildenstern (or is it Rosenkrantz?) is troubled by a fair coin that keeps coming up Heads. 
8. The challenge of writing down such a prior was posed by J. Smolin (private communication), Vaxjo, 2006.

9. C. M. Caves, C. A. Fuchs, and R. Schack, Studies in History and Philosophy of Modern Physics 38, 255-274 (2007). 